\title{
Spectral Analysis of Pressure, Noise and Vibration Velocity Measurement in Cavitation
}

\author{
Jana Jablonská ${ }^{\text {, Miroslav Mahdal }}{ }^{2}$, Milada Kozubková ${ }^{1}$ \\ ${ }^{1}$ Department of Hydromechanics and Hydraulic Equipment, Faculty of Mechanical Engineering, VŠB - Technical \\ University of Ostrava, 17. listopadu 15, 70833 Ostrava - Poruba, Czech Republic, jana.jablonska@vsb.cz \\ ${ }^{2}$ Department of Control Systems and Instrumentation, Faculty of Mechanical Engineering, VŠB, - Technical University of \\ Ostrava, 17. listopadu 15, 70833 Ostrava - Poruba, Czech Republic
}

\begin{abstract}
The article deals with experimental investigation of water cavitation in the convergent-divergent nozzle of rectangular cross-section. In practice, a quick and simple determination of cavitation is essential, especially if it is basic cavitation or cavitation generated additionally by the air being sucked. Air influences the formation, development and size of the cavity area in hydraulic elements. Removal or reduction of the cavity area is possible by structural changes of the element. In case of the cavitation with the suction air, it is necessary to find the source of the air and seal it. The pressure gradient, the flow, the oxygen content in the tank, and hence the air dissolved in the water, the air flow rate, the noise intensity and the vibration velocity on the nozzle wall were measured on laboratory equipment. From the selected measurements the frequency spectrum of the variation of the water flow of the cavity with cavitation without air saturation and with air saturation was compared and evaluated.
\end{abstract}

Keywords: Cavitation, noise, vibration, spectral analysis, CFD.

\section{INTRODUCTION}

Cavitation noise and vibrations result from the formation, growth and collapse of the cavitation bubbles. In particular, bubble collapse is accompanied by high flow velocities and high fluid pressure in the area of the cavitation bubbles, as a result of which compressive forces are induced [6], [9]. There are a large number of these bubbles in the cavitation area that interact with each other. According to literature [2], small cavitation bubbles have a frequency of their occurrence and extinction of up to $3 \mathrm{MHz}$, whereas large pulsating bubble structures (in the developed cavitation) can have a frequency of tens of Hz. This is used to identify cavitation in hydraulic elements. Appropriate location of sensors on the machine can determine the position of the cavity [3]. It is reported in the literature [1] that the origin of cavitation is characterized by frequencies ranging from $1 \mathrm{kHz}$ to $20 \mathrm{kHz}$, developed cavitation ranging from $20 \mathrm{~Hz}$ to $500 \mathrm{~Hz}$. In the literature [4], cavitation was detected at the frequency of $40 \mathrm{kHz}$ and it was proved that at lower frequencies the peak of the frequency spectrum of cavitation noise is of very little significance. Frequency values depend on the specific equipment and operating conditions.

The intensity of cavitation noise increases from the initial cavitation to fully developed cavitation and then decreases rapidly. Thus, the maximum noise intensity appears before fully developed cavitation. In addition, the maximum cavitation wear consistency with maximum cavitation noise [1] is evident. The increase of cavitation vibration, depending on the level of cavitation, is similar to that of cavitation noise, i.e. near the fully developed cavitation vibration is the maximum.

For the evaluation of noise, the sound pressure level in [dB] [5] is introduced, which is given by the relation

$$
L_{p}=20 \log \left(\frac{p}{p_{0}}\right)
$$

where $p$ is the sound pressure, $p_{0}=2 \cdot 10^{-5} \mathrm{~Pa}$ is the reference value of sound pressure. It is possible to use the acoustic intensity $I\left[\mathrm{~W} / \mathrm{m}^{2}\right]$, which is a vector defining the flow of acoustic energy per unit area in a direction perpendicular to this area. From this definition, its relation to the acoustic power $W[\mathrm{~W}]$ is

$$
W=\int_{S} I \cdot \mathrm{d} S
$$

Parallel to cavitation noise, cavitation phenomena cause device vibration. According to the literature [1], significant vibration frequencies range from $25 \mathrm{~Hz}$ to $1000 \mathrm{~Hz}$, while the other frequencies associated with cavitation bubble formation and disappearance are noticeable but with less amplitude. 


\section{EXPERIMENTAL AND MATHEMATICAL METHODS}

\section{Physical experiment}

Cavitation measurements were performed with the water flow in the convergent-divergent nozzle of rectangular cross section (CDN), its basic dimensions are shown in Fig.1.
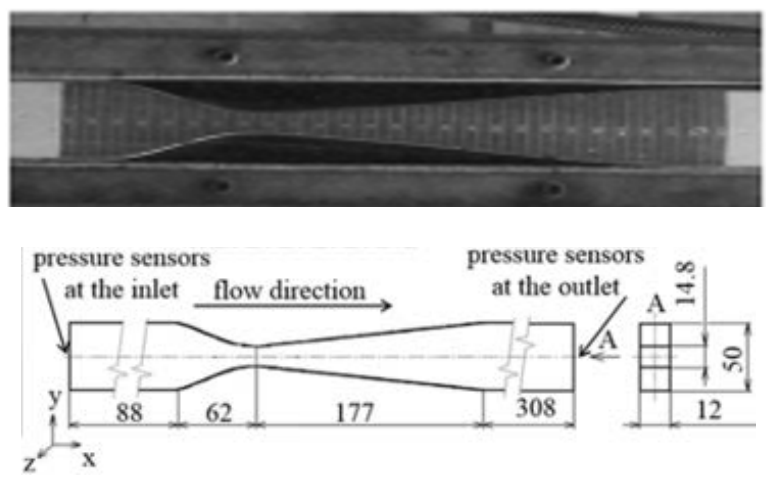

Fig.1. Photo and schema of convergent-divergent nozzle of rectangular cross section.

Cavitation was observed visually, by noise and vibration. The water flow was changed using a frequency converter from $12 \mathrm{~Hz}$ to $36 \mathrm{~Hz}$. The measurement range was limited by the structure and parameters of the equipment material. A number of measurements were also carried out on the flow of water with added air.

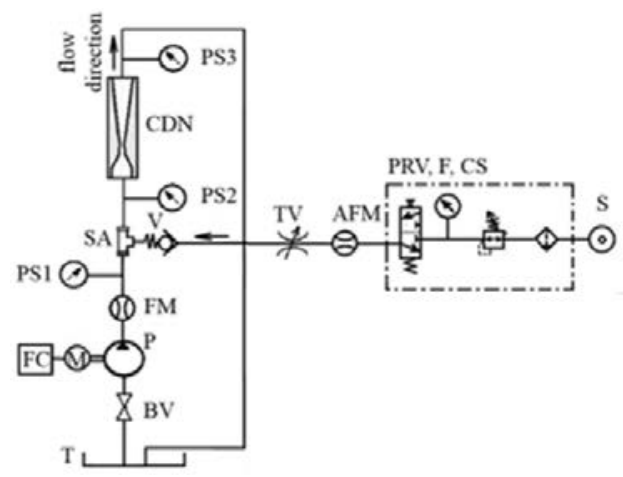

Fig.2. Measuring circuit $\mathrm{T}$ - tank, BV - ball valve, P- water pump, M - electric motor, FC - frequency converter, FM - inductive flowmeter, PS - pressure sensors, CDN - measured object convergent-divergent nozzle of rectangular cross-section, SA air saturation, $\mathrm{V}$ - one-way valve, TV - throttle valve to control air flow rate, AFM - air flowmeter, PRV, F, CS - pressure regulating valve with a filter unit and a condensate separator, $\mathrm{S}$ - compressed air source with a pressure gauge.

The scheme of measuring hydraulic circuit is evident from the diagram in Fig.2. The liquid (density $\rho=1001$ kg.m ${ }^{-3}$, viscosity $v=1.10^{-6} \mathrm{~m}^{2} \mathrm{~s}^{-1}$ ) is pumped into the circuit by a pump (P) which is connected to the tank (T) with water. A ball valve (BV) is located between the tank and the pump. The pump is driven by an electric motor (M) with speed control via a frequency converter (FC). An inductive flowmeter (FM) and a pressure gauge (PS1) are connected behind the pump. Attached air saturation device (SA) consists of air flow control valve (TV), air flowmeter (AFM), pressure control valve (PRV) with filter unit (F), condensate separator (CS), and compressed air source (S). The basic element of the circuit is the transparent convergent-divergent nozzle of the rectangular cross-section (CDN) to generate cavitation. Pressure sensors are placed before (PS2) and behind (PS3) the CDN nozzle. A 4507 B 004 accelerometer from Brüel \& Kjær is attached to the nozzle wall. The MiniSPL Noise Sensor is placed approximately $0.4 \mathrm{~m}$ in front of the nozzle. The Oxymax W COS61 water sensor was placed in the tank, which also sensed the temperature of the liquid. The result of the measurement was a signal directly proportional to the concentration of oxygen in the medium and the temperature of the medium. [7] The temperature of the medium did not change very much during the measurement, it was $25^{\circ} \mathrm{C}$.

Table 1. Measured values for variant I - without added air, II - with added air.

\begin{tabular}{|c|c|c|c|c|c|}
\hline & \multicolumn{4}{|c|}{ Variant I - without added air } \\
\hline $\begin{array}{c}f \\
{[\mathrm{~Hz}]}\end{array}$ & $\begin{array}{c}p_{2} \\
{[\mathrm{kPa}]}\end{array}$ & $\begin{array}{c}p_{3} \\
{[\mathrm{kPa}]}\end{array}$ & $\begin{array}{c}\Delta p \\
{[\mathrm{kPa}]}\end{array}$ & $\begin{array}{c}Q_{\mathrm{m}, \mathrm{w}} \\
{[\mathrm{kg} / \mathrm{s}]}\end{array}$ & $\begin{array}{c}C_{\mathrm{O} 2} \\
{[\mathrm{mg} / \mathrm{l}]}\end{array}$ \\
\hline 12 & 116 & 103 & 13 & 1.153 & 7.02 \\
\hline 14 & 122 & 107 & 15 & 1.380 & 7.03 \\
\hline 16 & 128 & 111 & 17 & 1.678 & 7.04 \\
\hline 18 & 136 & 115 & 21 & 1.920 & 7.04 \\
\hline 20 & 144 & 121 & 23 & 2.219 & 7.03 \\
\hline 22 & 154 & 126 & 28 & 2.408 & 7.03 \\
\hline 24 & 165 & 131 & 34 & 2.696 & 7.04 \\
\hline 26 & 184 & 137 & 47 & 2.838 & 7.05 \\
\hline 28 & 204 & 142 & 62 & 2.974 & 7.04 \\
\hline 30 & 225 & 147 & 78 & 3.269 & 7.02 \\
\hline 32 & 248 & 151 & 97 & 3.421 & 7 \\
\hline 34 & 273 & 160 & 113 & 3.673 & 6.94 \\
\hline 36 & 298 & 169 & 129 & 3.925 & 6.9 \\
\hline
\end{tabular}

\begin{tabular}{|c|c|c|c|c|c|c|}
\hline & & \multicolumn{5}{|c|}{ Variant II - with added air } \\
\hline $\begin{array}{c}f \\
{[\mathrm{~Hz}]}\end{array}$ & $\begin{array}{c}p_{2} \\
{[\mathrm{kPa}]}\end{array}$ & $\begin{array}{c}p_{3} \\
{[\mathrm{kPa}]}\end{array}$ & $\begin{array}{c}\Delta p \\
{[\mathrm{kPa}]}\end{array}$ & $\begin{array}{c}Q_{\mathrm{m}, \mathrm{w}} \\
{[\mathrm{kg} / \mathrm{s}]}\end{array}$ & $\begin{array}{c}C_{\mathrm{O} 2} \\
{[\mathrm{mg} / \mathrm{l}]}\end{array}$ & $\begin{array}{c}Q_{\mathrm{m}, \mathrm{air}, \mathrm{N}} \\
{[\mathrm{kg} / \mathrm{s}]}\end{array}$ \\
\hline 12 & 115 & 102 & 13 & 1.573 & 6.59 & $1.67 \cdot 10^{-5}$ \\
\hline 14 & 120 & 104 & 16 & 1.842 & 6.65 & $1.63 \cdot 10^{-5}$ \\
\hline 16 & 127 & 108 & 19 & 2.120 & 6.71 & $1.45 \cdot 10^{-5}$ \\
\hline 18 & 134 & 113 & 21 & 2.395 & 6.75 & $1.25 \cdot 10^{-5}$ \\
\hline 20 & 142 & 117 & 25 & 2.645 & 6.86 & $1.51 \cdot 10^{-5}$ \\
\hline 22 & 155 & 123 & 32 & 2.834 & 6.95 & $1.37 \cdot 10^{-5}$ \\
\hline 24 & 172 & 126 & 46 & 3.029 & 7.01 & $1.33 \cdot 10^{-5}$ \\
\hline 26 & 191 & 131 & 60 & 3.200 & 7.09 & $1.29 \cdot 10^{-5}$ \\
\hline 28 & 211 & 136 & 75 & 3.371 & 7.15 & $1.21 \cdot 10^{-5}$ \\
\hline 30 & 233 & 135 & 98 & 3.553 & 7.18 & $1.49 \cdot 10^{-5}$ \\
\hline 32 & 256 & 140 & 116 & 3.752 & 7.23 & $1.31 \cdot 10^{-5}$ \\
\hline 34 & 280 & 147 & 133 & 3.938 & 7.23 & $1.47 \cdot 10^{-5}$ \\
\hline 36 & 305 & 148 & 157 & 4.138 & 7.2 & $1.05 \cdot 10^{-5}$ \\
\hline
\end{tabular}


For each engine speed (i.e. flow rate) set, all relative pressures, noise intensity (MiniSPL Noise Sensor) and vibration velocities (4507 B 004 accelerometer) were recorded during the measurement on the computer with a given sampling frequency and subsequently evaluated. The tables show average pressure values measured by commonly available gauges with a time step $0.001 \mathrm{~s}$.

In Table 1. the measured average values of hydraulic variables $\left(p_{2}-\right.$ pressure before CDN, $p_{3}$ - pressure behind $\mathrm{CDN}, \Delta p=p_{2}-\mathrm{p}_{3}-$ pressure drop, $Q_{\mathrm{m}, \mathrm{w}}-$ water mass flow rate,

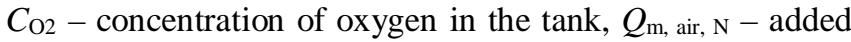
air mass flow rate under normal conditions) for variant without added air and variant with added air were evaluated.

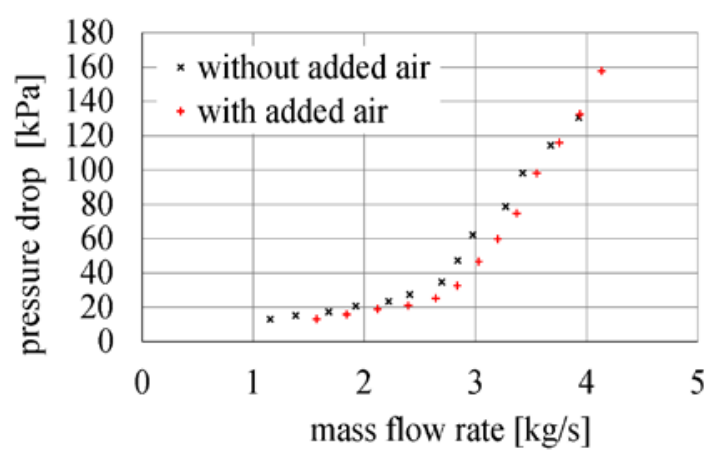

Fig.3. Dependence of pressure drop on mass flow rate for two variants of measurement.

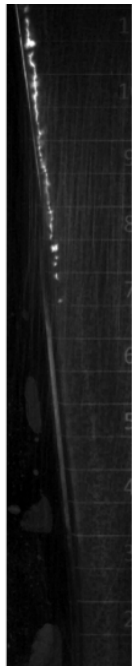

a)

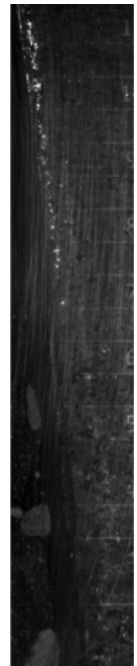

b)

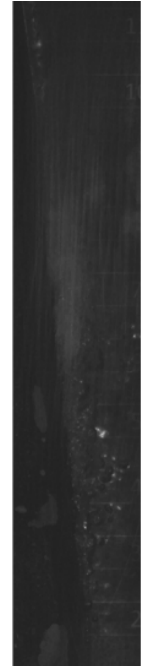

c)

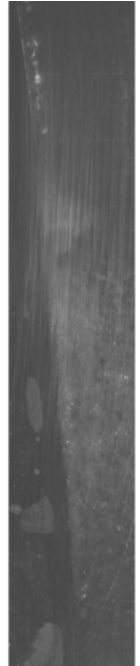

d)
Fig.4. Flowing without and with added air, without and with cavitation.

Fig.3. shows the dependence of the pressure drop on the CDN nozzle vs. the mass flow rate for both measured variants. A slower increase of the pressure drop in the lower flow area is in the non-cavitation flow. Then there is a sharp increase in the pressure gradient, which corresponds to the flow with cavitation (the flow is greater than $2.4 \mathrm{~kg} / \mathrm{s}$ ). Additionally, at a flow rate of up to $3.9 \mathrm{~kg} / \mathrm{s}$ with cavitation and no added air, the pressure drop on CDN is higher than that in variant II with added air. At higher flow rates and developed cavitation (flow rate is greater than $3.9 \mathrm{~kg} / \mathrm{s}$ ) it is the opposite, i.e. the pressure drop is higher in the case of flow with the added air than in the case of flow without added air. This phenomenon was also reported during further measurements.

The images of the CDN nozzle are illustrated in Fig.4., image a) presents a flow condition without cavitation and without added air, b) without cavitation and with added air, c) with cavitation and without added air, and d) with cavitation and with added air.

Initial cavitation was observed by sight and determined by noise. The basic assessment is usually performed using dimensionless parameters, i.e. dependence of Reynolds number $\quad \operatorname{Re}=\frac{v_{\max } d}{v} \quad$ versus cavitation number $\sigma=\frac{2\left(p_{\text {out }}-p_{\mathrm{n}}\right)}{\rho v_{\max }^{2}}$, see Fig.5. [8].

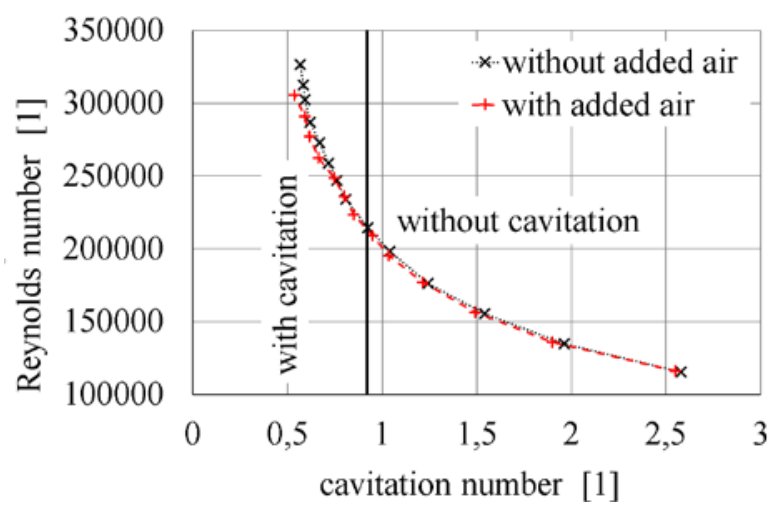

Fig.5. Reynolds number versus cavitation number ( $p_{\text {out }}-$ mean static pressure at the outlet from the nozzle, $p_{\mathrm{n}}$ - saturated pressure at given conditions, $\rho$ - density of water, $v$ - kinematic viscosity of water, $d-$ hydraulic diameter of the narrow cross section, $v_{\max }-$ velocity in narrow cross section).

In the case of variant I without added air, the initial cavitation was detected at higher engine revolutions (speeds) than $22 \mathrm{~Hz}$, i.e. with a cavitation number lower than 0.92 . The value of the cavitation number decreases with the added air. For the same Reynolds number, the cavitation number is higher for the variant with no added air. With the same cavitation number, the Reynolds number in the cavitation area is higher for the variant without added air. In an area without cavitation, the previous claim is unambiguous.

Fig.6. shows the air volume fraction at pressure near the bottom of the tank (i.e. $111.6 \mathrm{kPa}$ ) depending on the cavitation number. For variant I, the volume fraction of the dissolved air measured in the tank is plotted. For variant II, the sum of the volume fraction of the dissolved air in the tank and the volume fraction of the added air is shown. The volume fraction of the dissolved air in the tank was almost the same for both variants. The volume fraction of the added air into the system increases with the increasing cavitation number. 


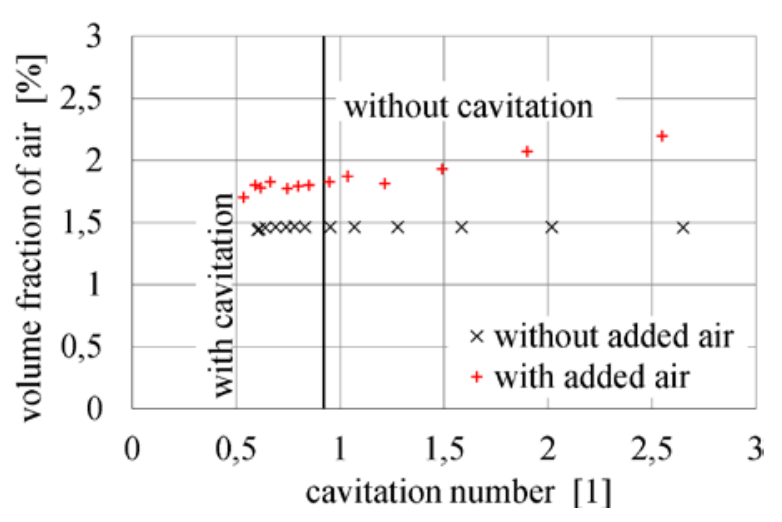

Fig.6. Volume fraction of air versus cavitation number.

Fig.7. shows the dependence of the loss coefficient on the cavitation number for both variants (variant I - without added air, variant II - with added air). Loss coefficient is defined by formula $\zeta=\frac{2 p_{2}}{\rho v_{\mathrm{in}}^{2}}, v_{\mathrm{in}}=\frac{4 Q_{\mathrm{m}, \mathrm{w}}}{\rho \pi d^{2}}$.

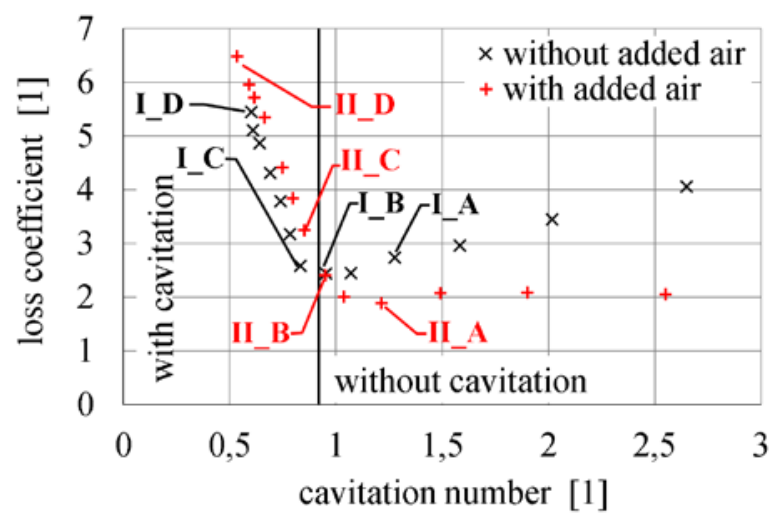

Fig.7. Loss coefficient versus cavitation number (marked variants will be solved later).

The loss coefficient in the cavitation-free flow area decreases with the decreasing cavitation number. In the cavitation flow area, the loss coefficient increases with the decreasing cavitation number. The lowest loss coefficient occurs in a critical cavitation number.

Marked variants in Fig.7. are further evaluated. Variant A represents flow without cavitation, $\mathrm{B}$ - flow at the cavitation interface, C - flow with initial cavitation, D - flow with fully developed cavitation.

\section{Mathematical modeling}

A 3D simulation of flow for variant I (considering air released from the fluid and no added air) was conducted with a multiphase turbulent RNG k- $\varepsilon$ model [10], [11], [12], [15]. The boundary condition at the input was defined by the mass flow rate of water, vapor and possibly air, at the output as pressure outlet. Mass flow rates and pressure values for boundary conditions were determined as average values from measurements.

\section{Results}

\section{Evaluation of the frequency spectrum of the measured pressure}

In Fig.8. and Fig.9. the frequency spectra from the measured pressures at the inlet to the CDN nozzle (i.e., pressure $p_{2}$, see Table 1.) are evaluated. The frequency range is limited by the sampling frequency of the pressure. The pressure was scanned within 0.001 seconds, so the frequency is limited to $400 \mathrm{~Hz}$.

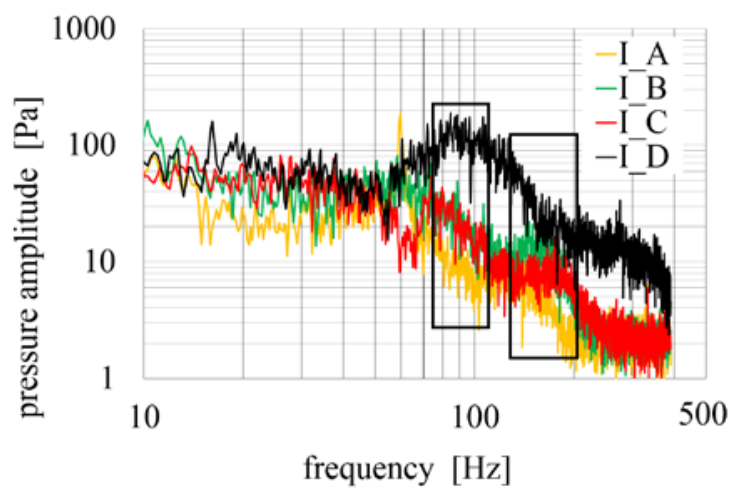

Fig.8. Pressure amplitude vs. frequency at inlet of the CDN nozzle, variant I - without the added air.

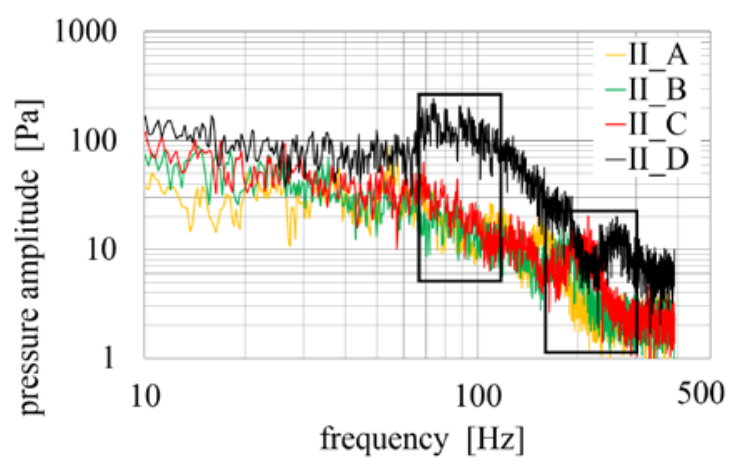

Fig.9. Pressure amplitude vs. frequency at inlet of the CDN nozzle, variant II - with the added air.

In variant $\mathrm{I}$ - for $\mathrm{D}$ there is a significant frequency around $87 \mathrm{~Hz}$, for A, B, C this frequency is not so pronounced. Significant frequencies for B, C, and D range from $151 \mathrm{~Hz}$ to $172 \mathrm{~Hz}$. The obtained frequencies specify the vortex structures due to the flow around the CDN nozzle and possibly cavitation.

In variant II - for all four measurements (with added air) there is a significant frequency in the range from $65 \mathrm{~Hz}$ to $75 \mathrm{~Hz}$. Other significant frequencies are in the range from $117 \mathrm{~Hz}$ to $158 \mathrm{~Hz}$, where it is noticeable that with increasing flow rate, this more significant frequency moves to the right. In addition, the frequencies are affected by the added air against variation $\mathrm{I}$.

In the fully developed cavitation of variants I_D and II_D, more pronounced frequencies of the vortex cavitation structures are evident. 
Evaluation of frequency spectrum of noise intensity and vibration velocity

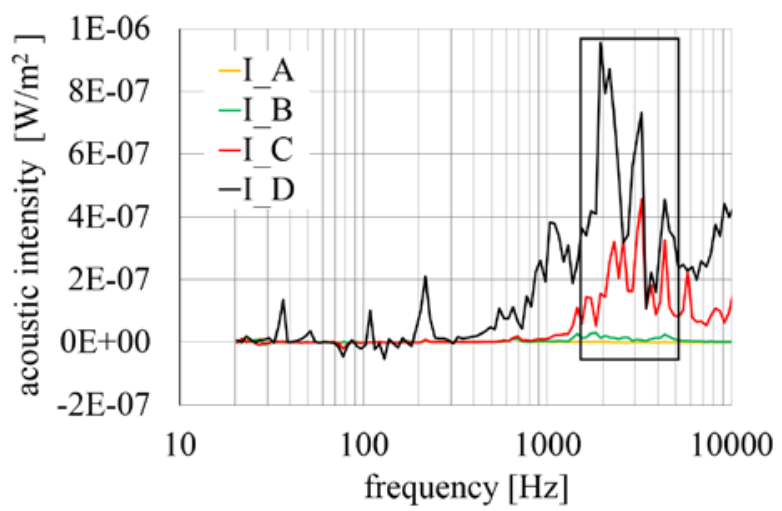

Fig.10. Acoustic intensity vs. frequency, variant I - without the added air.

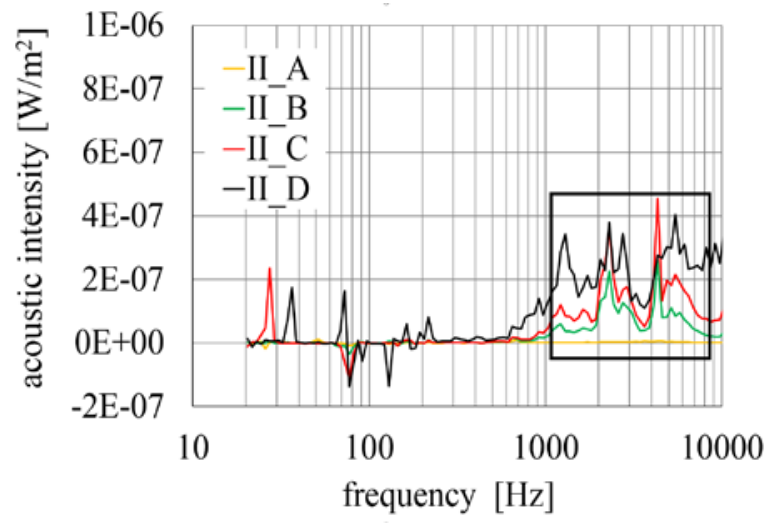

Fig.11. Acoustic intensity vs. frequency, variant II - with the added air.

Fig.10. and Fig.11. show the frequency spectra obtained from noise measurements. When measuring noise intensity, frequencies of less than $300 \mathrm{~Hz}$ are not considered, as they are influenced by the laboratory environment. The frequencies determining the formation and destruction of cavitation bubbles are significant. For variants I_A, I_B, and II_A (flow without cavitation), the amplitude of the noise intensity is very small. However, for variants I_C, II_B, and II_C, significant frequencies are $2300 \mathrm{~Hz}$ (vapor cavitation) and $4340 \mathrm{~Hz}$ (air cavitation). In variant I_D, the amplitudes of the noise intensity are more pronounced, since it is a fully developed cavitation, significant frequencies are in the range from $1900 \mathrm{~Hz}$ to $4300 \mathrm{~Hz}$. Variants II_D have significant frequencies in the range of $1300 \mathrm{~Hz}$ to $5500 \mathrm{~Hz}$. In case of variants II with added air there are significantly lower amplitudes of the noise intensity in the cavitation area, as the added air suppresses the noise intensity. Therefore, in terms of cavitation identification, noise measurement is not the most appropriate.

Variable frequency spectra of vibration velocity (Fig.12, Fig.13.) show low frequencies (up $500 \mathrm{~Hz}$ ) consistent with the pressure spectral analysis (see Fig.8., Fig.9.) and high frequencies that can be obtained from the records of the noise intensity (see Fig.10., Fig.11.).

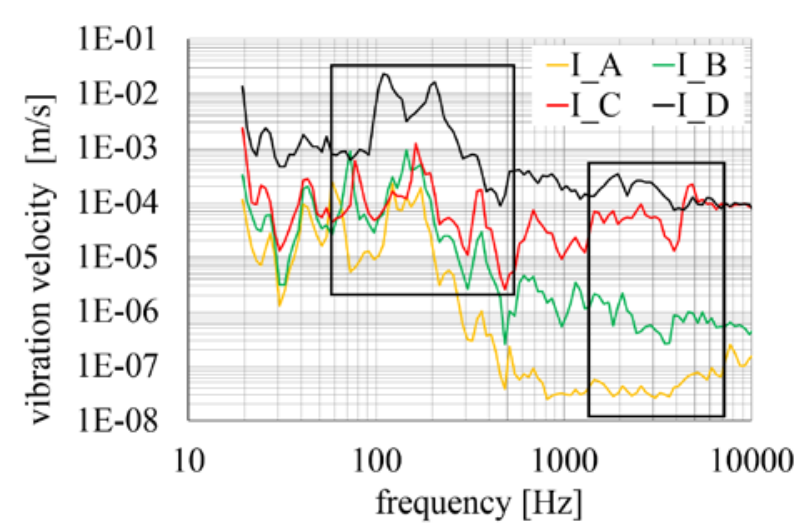

Fig.12. Vibration velocity vs. frequency, variant I - without the added air.

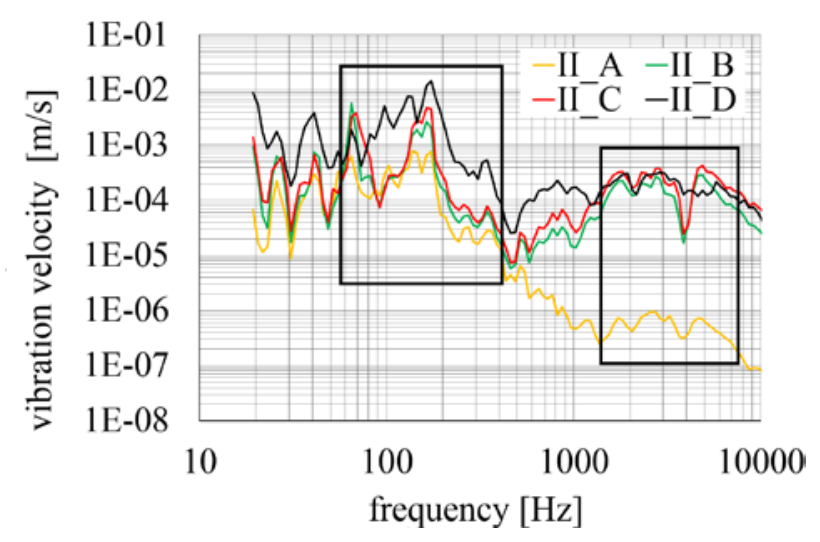

Fig.13. Vibration velocity vs. frequency, variant II - with the added air.

Approximately from $500 \mathrm{~Hz}$, the frequency spectrum of measurements I_A, I_B, and II_A is still decreasing (the turbulence effect) compared to other measurements, and the amplitude of the vibration velocity is very low. It is clear from this trend that cavitation does not occur in these measurements. The added air promotes cavitation even at lower flow rates and, consequently, higher frequencies (over $1000 \mathrm{~Hz}$ ) are better seen.

- Variant I - in the spectrum A, B, and C the significant frequencies ranging from $70 \mathrm{~Hz}$ to $90 \mathrm{~Hz}$, the frequencies ranging from $163 \mathrm{~Hz}$ to $172 \mathrm{~Hz}$ and around $365 \mathrm{~Hz}$ are evident. For spectrum D, there are three main frequencies $115 \mathrm{~Hz}, 217 \mathrm{~Hz}$, and $365 \mathrm{~Hz}$. The cavitation bubble generation and extinction frequencies are in the range from $2000 \mathrm{~Hz}$ to $5000 \mathrm{~Hz}$, while the spectrum amplitudes gradually increase with increasing flow rate.

Variant II - all spectra have a significant peak in the range of $65 \mathrm{~Hz}-70 \mathrm{~Hz}$, which characterize the vortex structures at the flow around the nozzle and all spectra have a significant peak in the range from $160 \mathrm{~Hz}$ to $170 \mathrm{~Hz}$ and also around $365 \mathrm{~Hz}$. Significant frequencies for B, C, and D are around $1940 \mathrm{~Hz}$ and $2900 \mathrm{~Hz}$, indicating the predominant vapor cavitation. For spectra B and C there is also a significant frequency around $5300 \mathrm{~Hz}$, but for $\mathrm{D}$ this frequency is shifted to $6100 \mathrm{~Hz}$. These frequencies indicate 
increased air cavitation. The amplitude of the spectra B, C, and $\mathrm{D}$ above $1000 \mathrm{~Hz}$ is approximately the same due to cavitation and the influence of added air.

Higher frequencies are approximately the same as those obtained from noise intensity measurements, but the amplitudes are not so pronounced.

\section{Mathematical modeling}

In Fig.14. the tested mathematical models are evaluated and compared with the measurement.

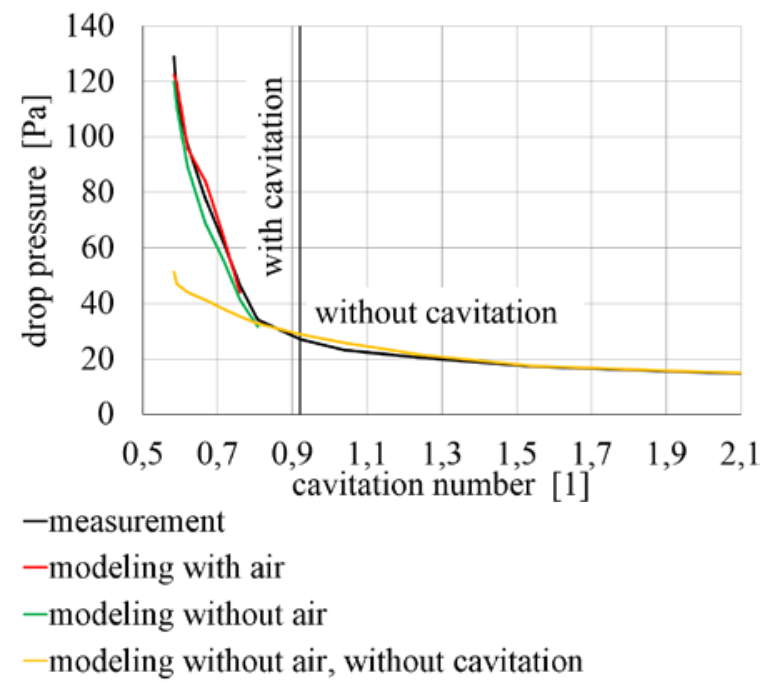

Fig.14. Pressure drop versus cavitation number for measurement and modeling variant.

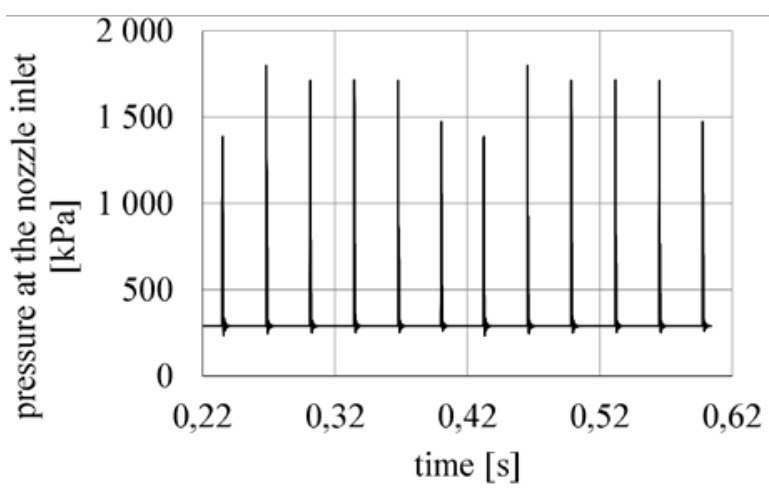

Fig.15. Record of pressure on CDN nozzle input obtained from mathematical modeling for variant I_D.

At first, the tasks (Fig.14., yellow line) were solved by the simplest stationary, two-phase (water, vapor), non-cavitation model. The deviation between the pressure drop and the measurement for the non-cavitation area ranged from 0 to $5 \%$, in the cavitation area the pressure drop deviation was up to $65 \%$. Therefore, it was necessary to use the cavitation Schnerr Sauer non-stationary model with a time step of $10^{-5} \mathrm{~s}$ in the area of cavitation flow due to the evaluation of the results by spectral analysis [14], [16], see Fig.14., green line. The deviation was up to $15 \%$. For further refinement, the model was extended to a cavitation three-phase model (water, vapor, air). In the inlet boundary condition, the mass air flow rate was defined as $0.1 \%$ of volume fraction. The deviation between the calculation and the measurement was within $5 \%$ (see Fig.14., red line). The value of the undissolved air released from the liquid in the tank has been estimated. The modeling methodology for the added air variant will be the same and will be tested in the future.

Fig.15. shows the record of pressure at the CDN nozzle input obtained from the modeling for variant I_D.

Subsequently, the frequency spectrum was evaluated from this record, see Fig.16. Significant frequencies in the box are identical to the significant frequencies gained from noise and vibration measurement.

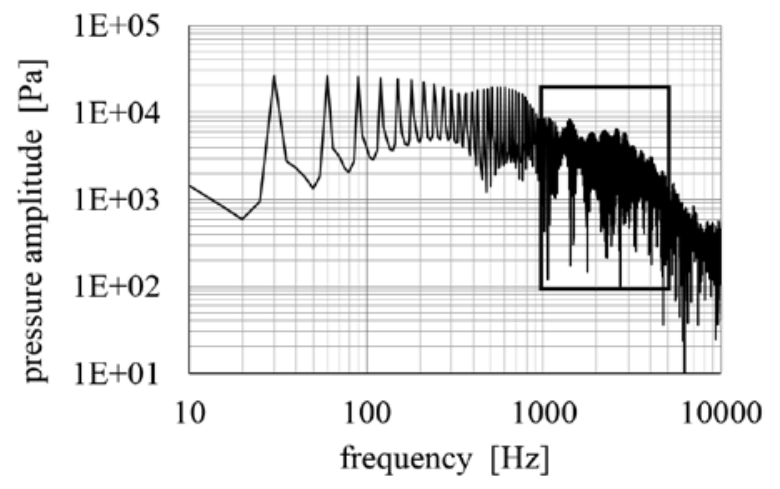

Fig.16. Pressure amplitude for variant I_D vs. frequency at CDN nozzle inlet (spectral analysis).

\section{DISCUSSION / CONCLUSIONS}

The article deals with the possibilities of detection of cavitation in hydraulic elements by means of spectral analysis determined by pressure, noise, and vibration measurement. The described methods are simple, cheap and fast. However, individual methods give results over a limited range of frequencies due to the characteristics of the gauges used.

Fig.17. shows all the frequency spectra (from measured inlet pressure, modeled input pressure, vibration velocity, and noise intensity) for variant I_D. The arrows show the previously described significant frequencies from the individual measurements. It can be clearly stated that to obtain information on the frequencies of the vortex structures with the developed cavitation in the case of flow through the nozzle, it is sufficient to measure the pressure by means of commonly used gauges. If it is necessary to obtain higher frequencies related to cavitation bubble formation and extinction, it is advisable to use noise intensity measurement. Measurement of vibration velocity gives sufficient information over the entire frequency range, but not so pronounced in amplitude. Mathematical modeling records the high frequencies associated with cavitation bubble formation and cessation, but a suitable model [10], [13], [14], the network, and the time step need to be chosen appropriately. Another disadvantage of mathematical modeling is the time consuming calculation. However, with newly developed elements, mathematical modeling is necessary. 


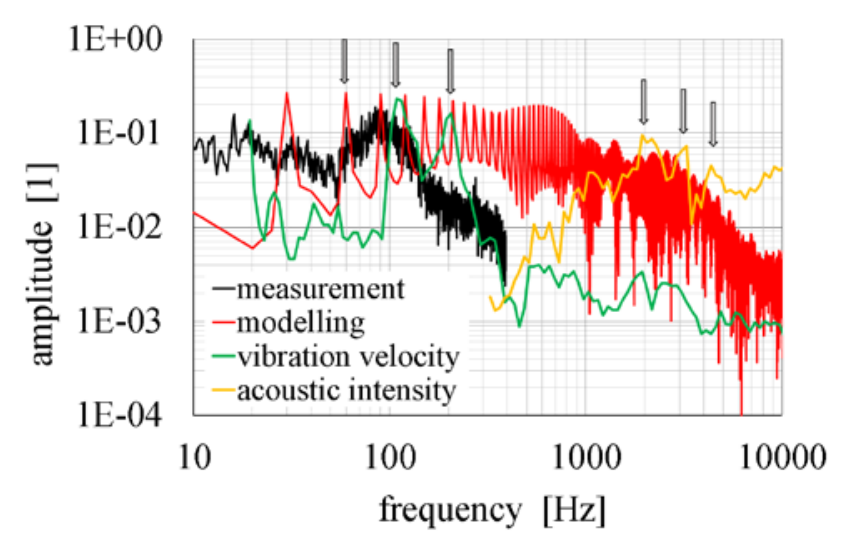

Fig.17. Spectral analysis of variant I_D.

The frequency spectrum obtained from the measured pressure compared to vibration velocity (liquid-plexiglass) and noise intensity (liquid-plexiglass-air transfer) shows a slight variation due to measurements in different environments.

Due to the measurement, an earlier indication of the initial cavitation in variant II_B was noted, indicating that the added air supports the formation and development of air cavitation and suppresses vapor cavitation. However, air cavitation does not have such a destructive effect on material as vapor cavitation. Vapor cavitation is more dangerous for hydraulic components.

When comparing the noise intensity for fully developed cavitation (variant D), it is clear that the added air suppresses the noise intensity amplitude and simultaneously suppresses vapor cavitation. This claim, however, is not apparent from the observation of the cavitation cloud with the naked eye.

From the thesis it follows that it will be necessary to deal with further mathematical methods for the theoretical mastering of cavitation.

\section{ACKNOWLEDGEMENT}

The work presented in this paper was supported by a grant SGS „Research on dynamics of fluid systems“. SP2017/103.

This work was supported by The Ministry of Education, Youth and Sports from the Large Infrastructures for Research, Experimental Development and Innovations project "Innovations National Supercomputing Centre LM2015070”.

\section{REFERENCES}

[1] Noskievič, J. (1990). Cavitation in Hydraulic Machinery and Equipment. Prague, Czech Republic: SNTL. (in Czech).

[2] Pearsall, I. S. (1966). Acoustic detection of cavitation. Proceedings of the Institution of Mechanical Engineers, Conference Proceedings, 181 (1).

[3] Brdička, M., Samek, L., Taraba, O. (1981). Cavitation: Diagnostics and Technical Use). Prague, Czech Republic: SNTL. (in Czech).
[4] Deeprose, W.M., King, N.W., McNulty, P.J. Pearsall, I.S. (1974). Cavitation noise, flow noise and erosion. In Proceedings of the Conference on Cavitation. London, UK: Institute of Mechanical Engineers, 373-381.

[5] Smetana, C. (1998). Noise and Vibration: Measurement and Evaluation. Prague, Czech Republic: Sdělovací technika. (in Czech)

[6] Brennen, Ch.E. (1995). Cavitation and Bubble Dynamics. Oxford University Press.

[7] Jablonská, J., Kozubková, M., Himr, D., Weisz, M. (2016). Methods of experimental investigation of cavitation in a convergent-divergent nozzle of rectangular cross section. Measurement Science Review, 16 (4), 197-204.

[8] Čarnogurská, M., Př́hoda, M., Popčáková, D. (2010). Modelling the flow conditions in the tunnel and its reduced model. Journal of Mechanical Science and Technology, 24 (12), 2479-2486.

[9] Meng, J., Liu, Z., An, K., Yuan, M. (2017). Simulation and optimization of throttle flowmeter with inner-outer tube element. Measurement Science Review, 17 (2), 6875.

[10] Knížat, B., Olšiak, R., Mlkvik, M. (2014). Observation of development of cavitation damage. EPJ Web of Conferences, 67, 02053.

[11] Ternik, P., Rudolf, R. (2013). Laminar natural convection of non-Newtonian nanofluids in a square enclosure with differentially heated side walls. International Journal of Simulation Modelling, 12 (1), 5-16.

[12] Sikora, R., Bureček, A., Hružík, L., Vašina, M. (2015). Experimental investigation of cavitation in pump inlet. EPJ Web of Conferences, 92, 02081.

[13] Jablonská, J., Bojko, M. (2015). Multiphase flow and cavitation - comparison of flow in rectangular and circular nozzle. EPJ Web of Conferences, 92, 02028.

[14] Schnerr, G.H., Sauer, J. (2002). Physical and numerical modeling of unsteady cavitation dynamics. In $4^{\text {th }}$ International Conference on Multiphase Flow (ICMF2001). Elsevier.

[15] Bureček, A., Hružík, L., Vašina, M. (2015). Determination of undissolved air content in oil by means of compression method. Strojniški vestnik Journal of Mechanical Engineering, 61 (7-8), 477-485.

[16] Bilus, I., Morgut, M., Nobile, E. (2013). Simulation of sheet and cloud cavitation with homogenous transport models. International Journal of Simulation Modelling, 12 (2), 94-106

Received August 19, 2017. Accepted November 13, 2017. 